\title{
Concurrent Passive Mode-Locked and Self- $Q$-Switched Operation in Laser Systems
}

\author{
J. Guo $\odot,{ }^{1,2}$ S. T. Cundiff $\odot,{ }^{1}$ J. M. Soto-Crespo, ${ }^{3}$ and N. Akhmediev ${ }^{4}$ \\ ${ }^{1}$ Department of Physics, University of Michigan, Ann Arbor, Michigan 48109, USA \\ ${ }^{2}$ Center of Ultra-Precision Optoelectronic Instrument Engineering, Harbin Institute of Technology, Harbin 150080, China \\ ${ }^{3}$ Instituto de Óptica CSIC, Serrano 121, 28006 Madrid, Spain \\ ${ }^{4}$ Department of Theoretical Physics, Research School of Physics, The Australian National University, \\ Canberra, Australian Capital Territory 2600, Australia
}

(Received 5 November 2020; accepted 19 April 2021; published 4 June 2021)

\begin{abstract}
Concurrent passive mode-locked and self- $Q$-switched operation of laser devices is modeled using the complex cubic-quintic Ginzburg-Landau equation. Experimental observations use a passively mode-locked fiber ring laser with a waveguide array as a fast saturable absorber. The shape of each individual self- $Q$ switched pulse and the periodic trains of pairs of such pulses are in a good qualitative agreement with the numerical results.
\end{abstract}

DOI: 10.1103/PhysRevLett.126.224101

Passive mode locking [1-3] and passive $Q$ switching [4-6] are two techniques often used for the generation of short pulses by solid-state, semiconductor, or fiber lasers. The most popular device for either mode locking or cavity finesse modulation is a nonlinear transmission element (saturable absorber) that modulates the losses in the cavity according to the light intensity. A variety of such devices has been suggested and used successfully in laser designs [7-13]. Passive mode locking produces pulses with durations from a few picoseconds to a few femtoseconds [14] with repetition rates typically in the mega- to gigahertz range, depending on the cavity round-trip time. On the other hand, the durations of the $Q$-switched pulses are on the micro- to nanosecond scale, while the repetition rate is in the kilohertz range. The latter is related to the speed of recovery of the population inversion. Both regimes of pulse generation can be actually realized in the same laser using the same nonlinear transmission device $[15,16]$. Moreover, $Q$-switched pulses may consist of a train of mode-locked pulses [15], which in a stationary regime of laser operation are dissipative solitons [17].

Dissipative solitons (DSs) are the result of a combined balance between dispersion, nonlinearity, gain, and loss in a laser cavity [18-20]. In many cases, this complex balance leads to a soliton of fixed shape. When emitted every round-trip, they generate a train of stable pulses with constant amplitude. However, this regime of pulse generation is not unique. It happens in a certain range of laser parameters. Out of this range, the soliton profile may change continuously from one round-trip to another, thus driving the laser to pulsating [21], breathing [22], and even stochastic [23] regimes. The change of the laser operational mode usually takes the form of a bifurcation. The new regime of operation is also stable but now the DS parameters oscillate periodically. Periods of oscillation may vary from two round-trips to hundreds $[21,22]$.
Periodic DS evolution is not necessarily a smooth process described by a sinusoidlike function. In a nonlinear system, the DS train envelopes can take the shape of longer pulses with sharp peak amplitudes [22,24,25]. These bursts of DS amplitude occupying many round-trips may occur periodically. In this case, each of these bursts can be considered as a self- $Q$-switched (SQS) pulse. Effectively, these bursts appear due to self-modulation of the cavity finesse influenced by the pulses circulating inside the cavity. In fact, if the short DS components cannot be resolved, only the envelope of these long pulses is recorded.

An important point that we make here is that the cubicquintic complex Ginzburg-Landau equation (CGLE) can describe both the passive mode-locking and the passive $Q$ switching regimes of pulse generation. Some examples, although implicitly, have been already presented [21,22]. Namely, the CGLE describes both fast effects of soliton formation in each round-trip and slow effects of soliton evolution from one round-trip to another that are caused by the nonlinear processes of self-management of the cavity finesse. This slow evolution resulted in a nearly sinusoidal change of the soliton parameters in many round-trips in prior studies [21]. In the present Letter, we observe also the periodic evolution of the soliton shape over many roundtrips, but it is far from being a simple function. Instead, we observe SQS pulse evolution with periodic sharp peaks. Moreover, we show that nonlinear effects may lead to period doubling of such SQS pulses.

Nontrivial effects of self- $Q$ switching predicted in our numerical simulations are confirmed experimentally using a fiber laser with a fast saturable absorber in the form of a waveguide array [26-28]. The generality of the results obtained with the CGLE model allows us to conclude that these effects may be observed in a wider range of laser 
systems that use fast saturable absorbers for short pulse generation.

For numerical simulations, we use as a model the CGLE, which in standard form reads [21]

$$
\begin{aligned}
i \psi_{z} & +\frac{D}{2} \psi_{\tau \tau}+|\psi|^{2} \psi+\nu|\psi|^{4} \psi \\
& =i \delta \psi+i \beta \psi_{\tau \tau}+i \epsilon|\psi|^{2} \psi+i \mu|\psi|{ }^{4} \psi,
\end{aligned}
$$

with $\psi$ being the envelope of the optical field, $D$ the averaged cavity dispersion, and $\nu$ the higher-order nonlinear coefficient. The right-hand side in Eq. (1) contains the dissipative terms with $\delta$ being the linear loss-gain parameter, $\epsilon$ representing the cubic gain-loss, and $\mu$ the gain saturation. $\beta$ is the spectral cavity bandpass coefficient. The $\tau$ variable represents the time within each round-trip moving with the pulse, while the $z$ variable is a continuous variable linearly proportional to the number of roundtrip times.

The model assumes that all processes in the cavity are fast. In particular, the passive mode-locking device does not have any time delay function. The coefficients $\delta, \epsilon$, and $\mu$ are influenced, among others, by this device. Despite its limitations, the CGLE proved itself as a useful model that describes, at qualitative level, many nontrivial processes occurring in laser systems. These include soliton explosions [29-31], dissipative soliton resonances [32,33], bifurcations of solitons [21,34], etc. The quintic terms in Eq. (1) are essential for a correct description of the dynamics of mode-locked lasers as first shown by Moores in 1993 [35].

Parameters in a continuous model are averaged over the length of the cavity. Methods for finding the correspondence between the averaged parameters of the CGLE and the actual parameters of a given cavity can be found elsewhere, for instance, in [36,37]. However, this can be only a very rough approximation. Additionally, doing so will reduce the generality of results that can be applied to other laser systems. Instead, we try to find empirically a set of the CGLE parameters that results in the same sequence of SQS pulses that are observed in our experiments. This requires thousands of numerical runs for scanning the multidimensional space of the equation parameters.

The pulse inside the fiber cavity is observed by coupling a small fraction of the circulating pulse train out of the cavity at one place in the cavity, thereby transforming a single DS circulating in the cavity into a train of pulses separated by the round-trip time $T$. The spectrum of such a pulse train consists of discrete comb lines that match the modes of a cavity when the phase evolution of the pulses is included [38]. We assume that the coupler losses are part of the overall losses in the cavity, which are taken into account in the CGLE parameters. In our experiments, $T=22 \mathrm{~ns}$. We denote the change of the $z$ variable in one round-trip as $\Delta z$. The value of $\Delta z$ can be roughly estimated through the number of DSs in one SQS pulse. Comparison with the experimental data below show that $\Delta z \approx 0.001$. The results of numerical simulations in Figs. 1(a) and 1(b) are presented as a function of time, which is the number of round-trips multiplied by $T$. The value of $\Delta z$ is not involved directly in the numerical simulations. It is an additional parameter in our approach necessary for adjusting numerical and experimental data. We use the step size in numerical simulations $10^{-6}$, i.e., significantly smaller than $\Delta z$.

Figure 1(a) shows the numerical results obtained for a chosen set of parameters that provides a SQS pulse shape close to the experimental one. Here, the linear loss $\delta=-0.1$, cubic gain $\epsilon=0.4$, quintic saturation of gain $\mu=-0.0005$, and spectral filtering $\beta=0.3$. The average cavity dispersion is $D=0.8$ and the quintic nonlinearity $\nu=0.01$. The shapes of two selected DS pulses with the minimum (blue dashed curve) and maximum (red curve) amplitudes in the train are shown in the inset of Fig. 1(a). The boundary conditions for the variable $\tau$ are zeros at the ends of the grid (far away from the DS). The total number of DSs (or round-trips) in Fig. 1(a) is around 108. As we can see, the output DSs evolve in the way that their envelope becomes a long SQS pulse with a nontrivial profile.
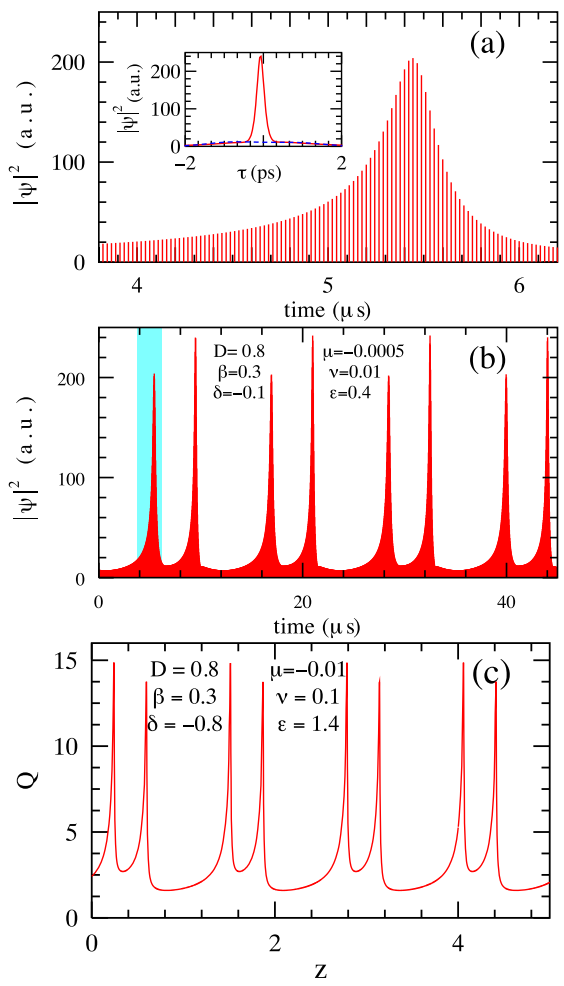

FIG. 1. (a) A sequence of DSs within a SQS pulse. The vertical axis corresponds to the maximal intensity $|\psi|^{2}$ of each DS. Inset: pulse profiles of the DS pulses at the maximum (red solid line) and minimum (blue dashed line) of the $Q$-switched pulse. (b) The same sequence in a longer timescale reveals the train of SQS pulses. The light blue area corresponds to the portion of the train shown in (a). (c) A sequence of SQS pulses for the set of parameters that are shown inside the figure. 
This is not the only revelation of these simulations. For the above set of parameters, two consecutive SQS pulses in the sequence are actually different. This can be seen from the same sequence in a larger timescale shown in Fig. 1(b). The latter includes nearly 2025 round-trips. Individual DS pulses cannot be resolved in this scale. We see a periodic train of longer pulses, where each period of the SQS train contains two different pulses. Importantly, we have observed similar pulse sequences in the experiments (see below).

In practice, it is difficult to measure the pulse intensity at its maximum when the pulse length is in pico- or femtosecond range. It is easier to deal with the total energy of such short pulses. The energy of a DS pulse generated within each round-trip is $Q(z)=\int_{-T / 2}^{T / 2}|\psi(z, \tau)|^{2} d \tau$, where $T$ is the round-trip time. We assume that the pulse is much shorter than $T$ and that its amplitude decays exponentially to zero at its tails. Then the actual value of $T$ is not essential and $Q(z)$ is the total energy per round-trip. For the self- $Q$ switching operation of the laser, we can calculate the average intensity of the train of DS pulses dividing the DS energy $Q$ by the duration of the round-trip time $T: I(z)=[Q(z) / T]=(1 / T) \int_{-T / 2}^{T / 2}|\psi(z, \tau)|^{2} d \tau$.

The value of $T$ is not explicitly present in the CGLE model. The energy $Q$ does not depend on $T$ as the tails of the soliton tend to zero. However, the average intensity $I(z)$ does depend on $T$. As in the experiment we are measuring the average intensity, this parameter must be equal to the actual round-trip time. On the other hand, the intensity in most of the experiments is normally presented in arbitrary units. Therefore, the actual value of $T$ does not influence the final results. Up to an arbitrary coefficient, the intensity of the $Q$-switched pulse is defined by the energy $Q$ of individual DSs. The latter is plotted in the results of numerical simulations below. For example, a sequence of SQS pulses similar to those in Fig. 1(b) rescaled to the $Q$ variable is shown in Fig. 1(c). Note that here we have used another set of parameters different from those in Fig. 1(b). Also, the horizontal axis is $z$, as the rescaling to time for this set of parameters may differ from the previous case.

Periodic sequences of SQS pulses similar to those in Fig. 1 can be observed for other sets of parameters, in a relatively large area in the CGLE parameter space. In order to show that, we have chosen a particular set of parameters admitting trains of SQS pulses and continuously varied the cubic gain parameter $\epsilon$. This way, we constructed the bifurcation diagram shown in Fig. 2(a). Here, the red solid dots correspond to the maxima of the SQS pulses in the train. In the interval $1.3<\epsilon \lesssim 1.45$, all pulses in the periodic train have the same amplitude. The bifurcation at $\epsilon \sim 1.45$ leads to two different SQS pulses per period. This transition from one pulse per period to two unequal pulses can be seen in Figs. 2(b) and 2(c). These plots show the pulse trains before [Fig. 2(b)] the bifurcation and after [Fig. 2(c)] the bifurcation point. The corresponding values

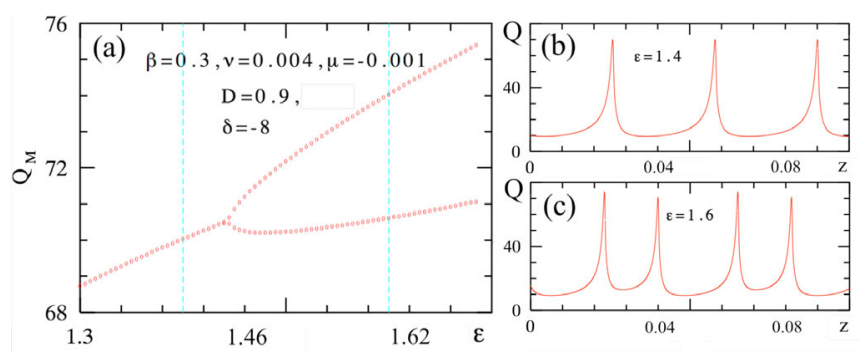

FIG. 2. (a) Bifurcation diagram for maximal $Q$ values of SQS pulses when cubic gain $\epsilon$ changes. The rest of parameters, shown within the plot, are fixed. The wave profiles for the two values of $\epsilon$ marked in (a) by dashed cyan vertical lines are shown in (b) and (c).

of $\epsilon$ are marked in Fig. 2(a) by the cyan dashed vertical lines. Two pulse separations within the period in Fig. 2(c) are also different. They are quite sensitive to the choice of the CGLE parameters.

For the experimental confirmation of the above results, we used a fiber ring laser with a simple design, shown in Fig. 3(a). The ring includes a standard telecommunication fiber (single mode fiber), an erbium-doped fiber, and a waveguide array (WGA) as a passive mode-locking device. WGAs were suggested for this purpose $[27,39]$ based on the fact that the transmission of the WGA depends on the intensity of light [26]. It is made of $\mathrm{AlGaAs}$ and has a design shown in Fig. 3(b). The WGA belongs to the category of "fast saturable absorbers" and perfectly satisfies the conditions for using the CGLE as a modeling tool. The advantages of the WGA over Kerr lensing or nonlinear polarization rotation are its engineerable saturation intensity and a quantified modulation depth.

The fundamental repetition rate of the cavity is $45.45 \mathrm{MHz}$. This corresponds to a round-trip time of $22 \mathrm{~ns}$. The transmission of the waveguide array at zero intensity is $27.6 \%$. The laser is pumped by a diode laser 1 at $976.0 \mathrm{~nm}$ with typical power of $750 \mathrm{~mW}$. The pump diode laser 2 operates at the same wavelength with a power of $600 \mathrm{~mW}$. The two pumps are combined with a fiber polarization beam combiner and then spliced to the $980 \mathrm{~nm}$ end of a wavelength division multiplexer. The splice loss is non-negligible.

Without the $40 \times$ objectives or WGA, the maximum output power is $4.24 \mathrm{~mW}$ from the $1 \%$ coupler. After inserting the waveguide array, the maximum output power is about $1.45 \mathrm{~mW}$. In the passive mode-locking regime of operation, the laser emits SQS pulses with a typical shape shown in Fig. 3(c). Each SQS pulse consists of around $\approx 112.5$ DS pulses that are not completely resolved in the scale of this figure. However, the maxima of these pulses are clearly seen in the plot. The designation of these pulses as DSs is confirmed by the optical spectrum, which is smooth and has a $3 \mathrm{~dB}$ bandwidth of approximately $2 \mathrm{~nm}$ that increases with increasing pulse energy. The number of DSs within the SQS pulse is roughly the same as in 

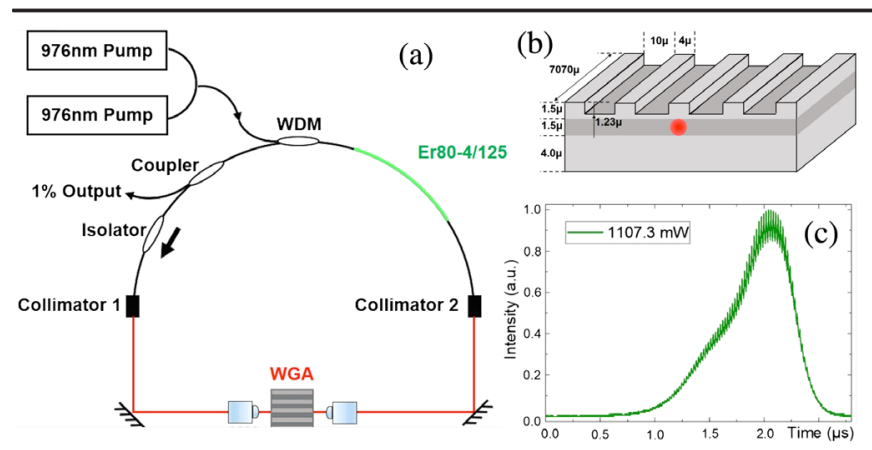

FIG. 3. Schematics of (a) experimental setup, (b) waveguide array, and (c) typical shape of a SQS pulse in the experiment.

Fig. 1(a). The pulse shape qualitatively is also similar to the profile shown in Fig. 1(a).

Experimental data provide further evidence for the validity of our theoretical model. Namely, we observed not isolated but periodic sequences of the SQS pulses similar to those in Figs. 1 and 2. Five examples of such sequences are shown in Fig. 4. The control parameter in these experiments is the pump power. It is shown in the upper right corner of each panel. Qualitatively, changing the pump power is equivalent to changing the cubic gain parameter $\epsilon$ in our numerical model.

Figure 4 shows the SQS pulse sequences before and after the bifurcation. The pulse separations and their relative amplitudes depend on the pump power. There is a qualitative correspondence between the pulse sequences in Figs. 2(b) and 2(c) and in Fig. 4. Namely, Fig. 4(a) has pulses with equal amplitudes and separations. This sequence is observed before the bifurcation point of transition from a single pulse per period to two pulses per period. Effectively, this point is a period doubling bifurcation for the SQS pulses. Figure 4(b) shows the pulse sequence after the bifurcation. The sequences in Figs. 4(c)-4(e) correspond to larger distances from the bifurcation point. The ratio of the two pulse amplitudes within the period is increasing from Fig. 4(b) to 4(e). This is the same qualitative behavior as in the bifurcation diagram in Fig. 2(a).

In conclusion, we have shown that SQS pulses can be modeled using the CGLE approach. For a certain set of parameters used in the CGLE, the pulse shapes and their sequences both in the theory and in the experiment are qualitatively similar. Our work shows that both the shape of a single SQS pulse and even the intricate periodic sequences of such pulses can be well described using our model. We have considered only the steady-state regimes of pulse generation. Any transitional effects have been avoided. The latter have been studied earlier in [16].

We stress that the self- $Q$ switching in our model is not related anyhow to the relaxation times of population inversion but entirely the result of nonlinear dynamics controlled by the fast saturable absorber. This way we can single it out from many other physical effects existing in the cavity. Clearly, any relaxation time can be added to the

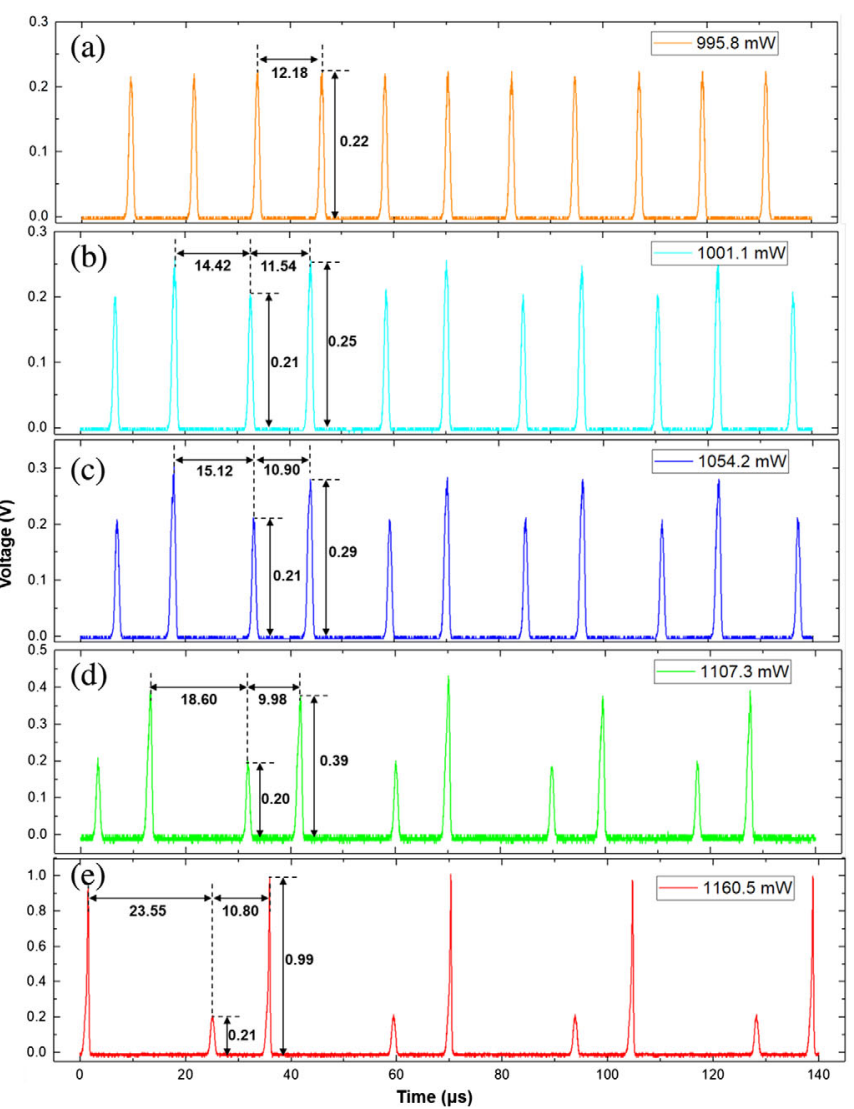

FIG. 4. Experimentally observed SQS pulses for five different pump powers, namely (a) $995.8 \mathrm{~mW}$, (b) $1001.1 \mathrm{~mW}$, (c) 1054.2 mW, (d) $1107.3 \mathrm{~mW}$ and (e) $1160.5 \mathrm{~mW}$. Timescales and relative intensities are shown in the figure. Individual DSs are not resolved in the scale of this plot.

model at later stages of studies of highly versatile $Q$ switching phenomena. They also influence the shape of DSs, as was shown in Ref. [40].

In this first work in this direction, we have restricted ourselves to a qualitative analysis. The detailed comparison of all the parameters in the theory to an even larger number of parameters in the experiment at this stage would be impossible. However, this qualitative analysis shows that the technique has a large potential in further extending the theory for describing even more complicated effects that might be observed in a large variety of existing laser systems.

J.G. acknowledges funding from the Chinese Scholarship Council (201706120188). The work of J.M. S. C. is funded by Spanish MICINN Grant No. RTI2018-097957-B-C33, and Comunidad de Madrid Grant No. S2018/NMT-4326 SINFOTON2-CM.

[1] E. P. Ippen, Appl. Phys. B, 58, 159 (1994).

[2] V. L. Kalashnikov, V. P. Kalosha, V. P. Mikhailov, I. G. Poloyko, and M. I. Demchuk, Opt. Commun. 109, 119 (1994). 
[3] R. Paschotta, R. Häring, A. Garnache, S. Hoogland, A. C. Tropper, and U. Keller, Appl. Phys. B 75, 445 (2002).

[4] J. A. Morris and C. R. Pollock, Opt. Lett. 15, 440 (1990).

[5] D. Popa, Z. Sun, T. Hasan, F. Torrisi, F. Wang, and A. C. Ferrari, Appl. Phys. Lett. 98, 073106 (2011).

[6] H. Li, H. Xia, C. Lan, C. Li, X. Zhang, J. Li, and Y. Liu, IEEE Photonics Technol. Lett. 27, 69 (2014).

[7] P. Wang, Y. Tang, H. Peng, G. Shi, T. He, H. Li, and Y. Liu, J. Mod. Opt. 64, 122 (2017).

[8] H. Ahmad et al., Laser Phys. Lett. 17, 095102 (2020).

[9] H. Hassan, M. A. Munshid, and A. Al-Janabi, Appl. Opt. 59, 1230 (2020).

[10] C. Feng, B. Ma, W. Qiao, G. Li, J. Zhao, K. Yang, D. Li, G. Li, S. Zhao, and T. Li, Appl. Opt. 59, 8066 (2020).

[11] E. Beyatl, F. Kaya, and H. Bilici, Appl. Opt. 59, 8247 (2020).

[12] J. Shang, J. Yang, H. Hao, Q. Li, L. Zhang, and J. Sun, Opt. Express 28, 22287 (2020).

[13] C. Y. Chen, Q. Wang, S. Huang, X. Zhang, K. X. Wang, M. W. Gao, and C. Q. Gao, Opt. Lett. 45, 3745 (2020).

[14] D. E. Spence, P. N. Kean, and W. Sibbett, Opt. Lett. 16, 42 (1991).

[15] C. Frerichs and U. B. Unrau, Opt. Fiber Technol. 2, 358 (1996).

[16] X. Liu, D. Popa, and N. Akhmediev, Phys. Rev. Lett. 123, 093901 (2019).

[17] P. Grelu and N. Akhmediev, Nat. Photonics 6, 84 (2012).

[18] N. Akhmediev and A. Ankiewicz, Dissipative Solitons, Lecture Notes in Physics Vol. 661 (Springer, Berlin, 2005).

[19] V. L. Kalashnikov, Phys. Rev. E 80, 046606 (2009).

[20] W. H. Renninger, A. Chong, and F. W. Wise, Phys. Rev. A 77, 023814 (2008).

[21] J. M. Soto-Crespo, M. Grapinet, Ph. Grelu, and N. Akhmediev, Phys. Rev. E 70, 066612 (2004).

[22] J. Peng, S. Boscolo, Z. Zhao, and H. Zeng, Sci. Adv. 5, eaax1110 (2019).
[23] D. Churkin, S. Sugavanam, N. Tarasov, S. Khorev, S. V. Smirnov, S. M. Kobtsev, and S. K. Turitsyn, Nat. Commun. 6, 7004 (2015).

[24] H. J. Kbashi, M. Zajnulina, A. G. Martinez, and S. V. Sergeyev, Laser Phys. Lett. 17, 035103 (2020).

[25] A. Khanolkar and A. Chong, Opt. Lett. 45, 6374 (2020).

[26] H. S. Eisenberg, Y. Silberberg, R. Morandotti, A. R. Boyd, and J. S. Aitchison, Phys. Rev. Lett. 81, 3383 (1998).

[27] J. L. Proctor and J. N. Kutz, Opt. Lett. 30, 2013 (2005).

[28] Q. Chao, D. D. Hudson, J. Kutz, and S. T. Cundiff, IEEE Photonics J. 4, 1438 (2012).

[29] S. T. Cundiff, J. M. Soto-Crespo, and N. Akhmediev, Phys. Rev. Lett. 88, 073903 (2002).

[30] A. F. J. Runge, N. G. R. Broderick, and M. Erkintalo, Optica 2, 36 (2015).

[31] O. Descalzi, C. Cartes, J. Cisternas, and H. R. Brand, Phys. Rev. E 83, 056214 (2011).

[32] W. Chang, A. Ankiewicz, J. M. Soto-Crespo, and N. Akhmediev, Phys. Rev. A 78, 023830 (2008).

[33] Q. Gong, H. Zhang, D. Deng, and J. Zu, IEEE Photonics J. 12, 1502708 (2020).

[34] D. Gomila, A. J. Scroggie, and W. J. Firth, Physica (Amsterdam) 227D, 70 (2007).

[35] J. D. Moores, Opt. Commun. 96, 65 (1993).

[36] A. Komarov, H. Leblond, and F. Sanchez, Phys. Rev. E 72, 025604(R) (2005).

[37] A. I. Korytin, A. Yu. Kryachko, and A. M. Sergeev, Radiophys. Quantum Electron. 44, 428 (2001).

[38] S. T. Cundiff, J. Phys. D 35, R43 (2002).

[39] D. D. Hudson, K. Shish, T. R. Schibli, J. N. Kutz, D. N. Christodoulides, R. Morandotti, and S. T. Cundiff, Opt. Lett. 33, 1440 (2008).

[40] N. N. Rozanov, S. V. Fedorov, and G. V. Khodova, Opt. Spectrosc. 88, 790 (2000). 